\title{
Optically-Triggered Nanoscale Memory Effect in a Hybrid Plasmonic-Phase
}

\section{Changing Nanostructure}

\author{
Dang Yuan Lei ${ }^{\dagger, *}$, Kannatassen Appavoo ${ }^{\ddagger}$, , Filip Ligmajer ${ }^{\dagger, \S}$, Yannick Sonnefraud \\ Richard F. Haglund, Jr ${ }^{\ddagger, l}$ and Stefan A. Maier ${ }^{\#}$ \\ ${ }^{\dagger}$ Department of Applied Physics, The Hong Kong Polytechnic University, Hong Kong, China. \\ ${ }^{\ddagger}$ Interdisciplinary Materials Science Program, Vanderbilt University, Nashville, TN 37235-0106, USA. \\ ${ }^{\perp}$ Centre for Functional Nanomaterials, Brookhaven National Laboratory, Upton, NY11973, USA. \\ ${ }^{\S}$ Central European Institute of Technology, Brno University of Technology, Technická 10, 61669 Brno, \\ Czech Republic. \\ "Institut Néel, CNRS UPR2940, 25 rue des Martyrs BP166, 38042 Grenoble Cedex 9, France. \\ "Department of Physics and Astronomy, Vanderbilt University, Nashville, TN 37235-1807, USA. \\ ${ }^{\#}$ Department of Physics, Imperial College London, London SW7 2AZ, UK.
}

\begin{abstract}
Nanoscale devices - such as all-optical modulators and electro-optical transducers - can be implemented in heterostructures that integrate plasmonic nanostructures with functional active materials. Here we demonstrate all-optical control of a nanoscale memory effect in such a heterostructure by coupling the localized surface plasmon resonance (LSPR) of gold nanodisk arrays to a phase-changing material $(\mathrm{PCM})$, vanadium dioxide $\left(\mathrm{VO}_{2}\right)$. By latching the $\mathrm{VO}_{2}$ in a distinct correlated metallic state during the insulator-to-metal transition (IMT), while concurrently exciting the hybrid nanostructure with one or more ultraviolet optical pulses, the entire phase space of this correlated state can be accessed optically to modulate the plasmon response. We find that the LSPR modulation depends strongly but linearly on the initial latched state, suggesting that the memory effect encoded in the plasmon resonance wavelength is linked to the strongly correlated electron states of the $\mathrm{VO}_{2}$. The continuous, linear variation of the electronic and optical properties of these model heterostructures opens the way to multiple design strategies for hybrid devices with novel optoelectronic functionalities, which can be controlled by an applied electric or optical field, strain, injected charge or temperature.
\end{abstract}


Keywords: surface plasmons; metal nanoparticles; vanadium dioxides; plasmonic memory effect; phase transformation 
Metallic nanostructures support localized surface plasmons (LSPs) and surface-plasmon polaritons (SPPs), coherent oscillations of conduction electrons at metal-dielectric interfaces. ${ }^{1}$ Surface plasmon excitations occur in ultrasmall mode volumes, generating high local electric fields in passive nanophotonic devices ranging from plasmon waveguides to plasmon lenses. ${ }^{2,3}$ The optical response of plasmonic structures can be controlled by electro-optical, ${ }^{4-6}$ thermaloptical $^{7-9}$ or magneto-optical properties ${ }^{10-13}$ of functional materials in close proximity. All-optical modulation of SPP propagation in metal films ${ }^{14-15}$ and of LSPs in metal nanostructures ${ }^{16-19}$ is also well known. In all these schemes, however, the modulated plasmon response is necessarily transient; the metal nanostructures revert to their initial plasmonic states as soon as the external stimulus is removed. On the other hand, in many applications it would be desirable to retain functionality in a plasmonic device even after the triggering signal disappeared, allowing tunable, persistent modulation of plasmonic properties. Accessing multiple plasmonic states linearly and repeatedly by active control of plasmon modes could enhance functionality in optoelectronic devices $^{20}$ and enable on-chip integration of optical and electronic functionalities.

Phase-change materials (PCMs) - already widely used in rewritable optical data storage and non-volatile electronic memory ${ }^{21}$ - offer unique possibilities for persistent, reversible tuning of surface plasmons by combining PCMs with metal nanostructures to create new or multiple plasmonic functionalities. A promising PCM for this purpose is vanadium dioxide $\left(\mathrm{VO}_{2}\right)$ with its strong correlations among orbital, spin and lattice degrees of freedom. A modest external stimulus can switch $\mathrm{VO}_{2}$ through non-congruent electronic (from insulating to metallic) and structural (from M1 monoclinic to $\mathrm{R}$ rutile) phase transformations. When $\mathrm{VO}_{2}$ is heated, this insulator-to-metal transition (IMT) occurs near a critical temperature $\left(T_{\mathrm{c}}\right)$ of $68{ }^{\circ} \mathrm{C}$ in bulk crystals $^{22}$ and thin films. ${ }^{23}$ The IMT can also be induced mechanically, ${ }^{24}$ electrically, ${ }^{25}$ optically ${ }^{26}$ 
and by charge injection, ${ }^{27}$ enabling active modulation of LSPs and SPPs in metallic nanostructures integrated with $\mathrm{VO}_{2} \cdot{ }^{28-31}$

Although it is generally understood how plasmons are modulated in hybrid nanostructures switched between the fully insulating and fully metallic states,$^{32}$ the potential for exploiting the entire strongly correlated phase space of a PCM has never been demonstrated at the nanoscale. In this paper, we show how the plasmonic state of a model hybrid nanostructure can be controlled by sampling different initial states within the region of strong electron correlations. We demonstrate persistent optical modulation of localized surface plasmon resonance (LSPR) in a model heterostructure comprising Au nanodisks lithographically patterned on a thin $\mathrm{VO}_{2}$ film. Throughout the temperature window of the IMT, the $\mathrm{VO}_{2}$ can be latched thermally in the selected strongly correlated metallic state. ${ }^{33}$ Because of the hysteretic response of the first-order $\mathrm{VO}_{2}$ phase transition, the LSPR can be persistently tuned by successive ultraviolet (UV) light pulses as long the system remains in the latched, initial state. This dynamic is highly repeatable, lasts for several hours and persists as long as the external stimulus is not varied. ${ }^{34-35}$ Hence the entire phase-space of this PCM can be optically addressed when the hybrid nanomaterial is latched at different starting temperatures during the IMT.

More interestingly, the subsequent temporal evolution of the LSPR depends strongly on the initial temperature from which the system is cooled, implying that the LSPR "memorizes" the initial state prepared in the hybridized plasmonic nanostructure and the strongly correlated electron state in the $\mathrm{VO}_{2}$. It is crucial to note that here the temperature is simply a proxy for the parameter that describes the initial, latched state of the $\mathrm{VO}_{2} \mathrm{PCM}$. Known properties of $\mathrm{VO}_{2}$ suggest, therefore, that the novel resonant memory effect described here should be observable 
regardless of whether the initial state is prepared by optically, electrically or mechanically initializing the hybrid plasmonic-phase-changing heterostructure.

\section{Mechanism of persistent tuning of a "memplasmon" resonance.}

Figure 1 shows the hybrid plasmonic-phase-change optical material, comprising periodic arrays of $\mathrm{Au}$ nanostructures on a thin $\mathrm{VO}_{2}$ film (see Figure 1a), along with a schematic of the mechanism for persistently tuning the LSPR in Au nanostructures interacting with the strongly correlated metallic state of the $\mathrm{VO}_{2}$. When the $\mathrm{VO}_{2}$ layer is initialized in the strong-correlation regime (shaded area, Figure 1b) by setting the temperature, each successive UV-light pulse transforms more crystalline nanodomains of the $\mathrm{VO}_{2}$ into the metallic state without recovering the initial state, manifested by a continuous change in optical transmission. This yields persistent tuning of the resonant plasmon response of $\mathrm{Au}$ nanostructures on top of the $\mathrm{VO}_{2}$ film, unlike non-phase-changing, active plasmonic systems with transient resonance modulation (Figure 1c).
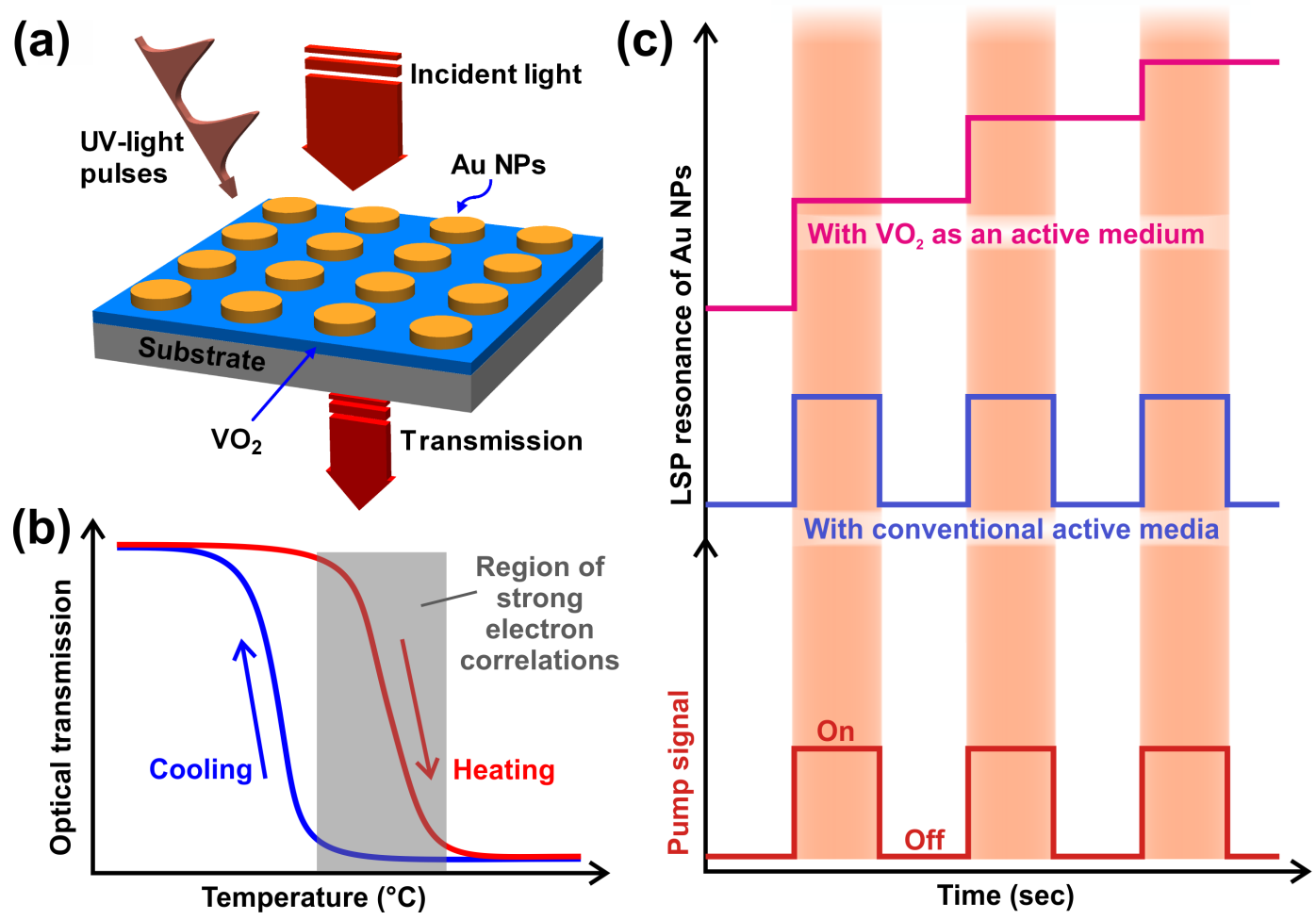
Figure 1: Model for persistent tuning of a "memplasmon" resonance. (a) Experimental schematic for the heating cycle of the phase transition: (a) Array of periodic Au nanodisks fabricated lithographically on a thin $\mathrm{VO}_{2}$ film deposited on a $\mathrm{SiO}_{2}$ substrate. Optical extinction measurements are conducted while successive UV-light pulses pump the $\mathrm{VO}_{2}$ film into new states. The device is mounted on a temperature-controlled stage. (b) Hysteretic response in the optical transmission of $\mathrm{VO}_{2}$, showing the region of strong electron-electron correlations during the heating cycle of the phase transition (shaded gray area). (c) Schematic comparison of plasmon resonance modulation schemes with conventional active media (blue) and the phasetransforming $\mathrm{VO}_{2}$ (pink) when pumped by successive optical pump pulses (red). The strongly correlated electron state in the $\mathrm{VO}_{2}$ is initialized and maintained by setting substrate temperature.

\section{Sample fabrication and large modulation of LSPR via phase transformation.}

Thin $\mathrm{VO}_{2}$ films with nominal thickness of $50 \mathrm{~nm}$ were fabricated on glass substrates by pulsed laser ablation of a vanadium target in 250 mTorr oxygen. Subsequent annealing of the samples at $450{ }^{\circ} \mathrm{C}$ for 40 minutes in 10 mTorr of oxygen rendered the film stoichiometric, crystalline and switching. A complete description of the protocol for characterizing optical response and crystal structure can be found elsewhere. ${ }^{23}$ Four different arrays of $\mathrm{Au}$ nanodisks were fabricated by electron-beam lithography on the $\mathrm{VO}_{2}$ film; the nominal diameter $\mathrm{D}$ of the $\mathrm{Au}$ nanostructures was varied from $80 \mathrm{~nm}$ to $210 \mathrm{~nm}$ while keeping thickness constant at $25 \mathrm{~nm}$. The Au nanodisks were arranged on a square lattice with a pitch of $500 \mathrm{~nm}$, shorter than the wavelength of interest (above $700 \mathrm{~nm}$ ) in order to avoid diffraction effects in the transmission measurements. Figure 2a shows typical SEM images of the Au nanostructures, exhibiting reproducibility over a large area.

Extinction spectra of the arrays, defined as (1 - Transmission), were acquired in an inverted optical microscope (Bruker Hyperion 2000) integrated with a Fourier-transform infrared spectrometer. ${ }^{30}$ Extinction spectra were measured while a Peltier thermoelectric element mounted on the sample stage cycled the $\mathrm{VO}_{2}$ film through its phase transition by heating and cooling 
through a set temperature program. The external temperature was maintained within $\pm 0.1{ }^{\circ} \mathrm{C}$ over the entire measurement cycle in all experiments. Figure $2 \mathrm{~b}$ shows the extinction spectra of three $\mathrm{Au}$ nanostructure arrays acquired below $\left(\mathrm{T}=25{ }^{\circ} \mathrm{C}\right.$, black curves $)$ and above $\left(\mathrm{T}=75{ }^{\circ} \mathrm{C}\right.$, red curves) the insulator-to-metal transition of $\mathrm{VO}_{2}$ and reveals that the LSPR of $\mathrm{Au}$ nanodisks experiences a substantial blue-shift due to the IMT.

The shift in LSPR for each array is plotted as a function of nanostructure diameter in Figure 2c, and shows that the larger Au nanostructures also exhibit larger resonance shifts because the dielectric contrast in $\mathrm{VO}_{2}$ during its phase transformation increases with wavelength, ${ }^{36}$ further shifting the LSPR. In addition, the evanescent near-fields associated with the larger Au nanodisks sample a larger interaction volume in the phase-transforming $\mathrm{VO}_{2}$ film. The ensemble of Au nanodisks is distributed over a $\mathrm{VO}_{2}$ film having a broad range of grain sizes, each of which switches at a statistically determined temperature. ${ }^{37}$ Hence the average LSPR tuning should be the same for each nanostructure array and is expected to be a function of the effective or meanfield fraction of metallic phase in the underlying polycrystalline $\mathrm{VO}_{2}$ layer. 

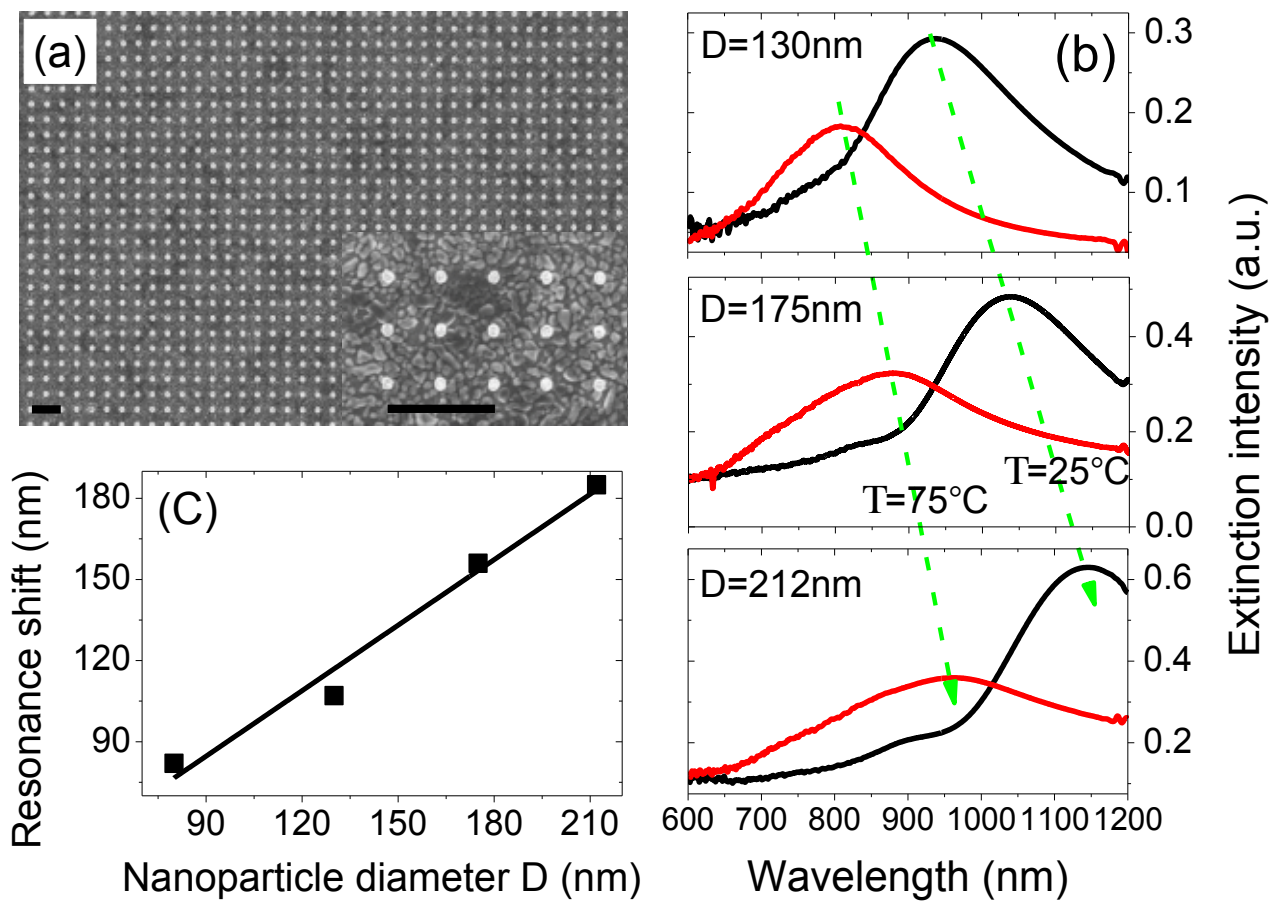

Figure 2: Modulation of localized surface plasmon resonances. (a) Plan-view SEM image of $\mathrm{Au}$ nanostructures patterned on a 50-nm thick $\mathrm{VO}_{2}$ film. Inset: Nanostructure array at higher magnification. Scale bars are $1 \mu \mathrm{m}$ in each case. (b) Measured extinction spectra of $\mathrm{Au}$ nanostructures for three diameters $D$ at room- (black) and high-temperature (red). (c) Plasmon resonance peak shift due to the phase transformation of $\mathrm{VO}_{2}$ film as a function of nanostructure diameter (black squares), with linear fit (black line).

\section{Hysteretic variation of localized surface plasmon resonance.}

We next investigate the changes in the LSPR in $\mathrm{Au}$ nanostructures during the phase transformation of $\mathrm{VO}_{2}$ by measuring extinction spectra for the array of 130-nm diameter $\mathrm{Au}$ nanodisks while reversibly cycling the $\mathrm{VO}_{2}$ film across the strong-correlation regime $\left(30^{\circ} \mathrm{C}\right.$ $80^{\circ} \mathrm{C}$ ) with a resolution as small as $0.5^{\circ} \mathrm{C}$. The measured extinction spectra in Figure $3 \mathrm{a}$ for the hybrid system show smooth transitions in both resonance wavelength and LSPR intensity. By fitting the extinction spectra, we can map the hysteresis in resonance wavelength of the Au LSPR as a function of substrate temperature (Figure 3b). The hystereses of the Au LSPR and the far- 
field transmission of a bare $\mathrm{VO}_{2}$ film (50 nm thick, Figure 3d) exhibit similar widths. The close similarity between the two hysteresis curves indicates that the evolution of the Au LSPR tracks the phase-transition properties of the underlying $\mathrm{VO}_{2}$ film and can properly be used as an optical marker for the change in local optical properties in the phase-changing material. The slight reduction in thermal hysteresis width $\Delta \mathrm{T}$ for the LSPR compared to that for the bare $\mathrm{VO}_{2}$ film can be attributed to the presence of additional nucleation sites at the $\mathrm{Au} / \mathrm{VO}_{2}$ interface, which facilitate the phase-transformation of $\mathrm{VO}_{2}$ by reducing the phase-transition barrier energy. ${ }^{38}$

To further correlate the LSPR modulation with the phase changing dynamics of $\mathrm{VO}_{2}$, Figures $3 \mathrm{c}$ and $3 \mathrm{e}$ show respectively the plasmon resonance wavelength of the Au nanodisks and the transmission intensity of the bare $\mathrm{VO}_{2}$ film as a function of the $\mathrm{VO}_{2}$ metallic fraction that is determined from the hysteretic parameters shown in Figures $3 \mathrm{~b}$ and $3 \mathrm{~d}$ by using the method described in Ref. 48. The results demonstrate a linear relationship between both plasmon resonance wavelength and transmission intensity, and the metallic fraction of $\mathrm{VO}_{2}$, thereby indicating a continuous, linear variation of the electronic and optical properties of both systems. 

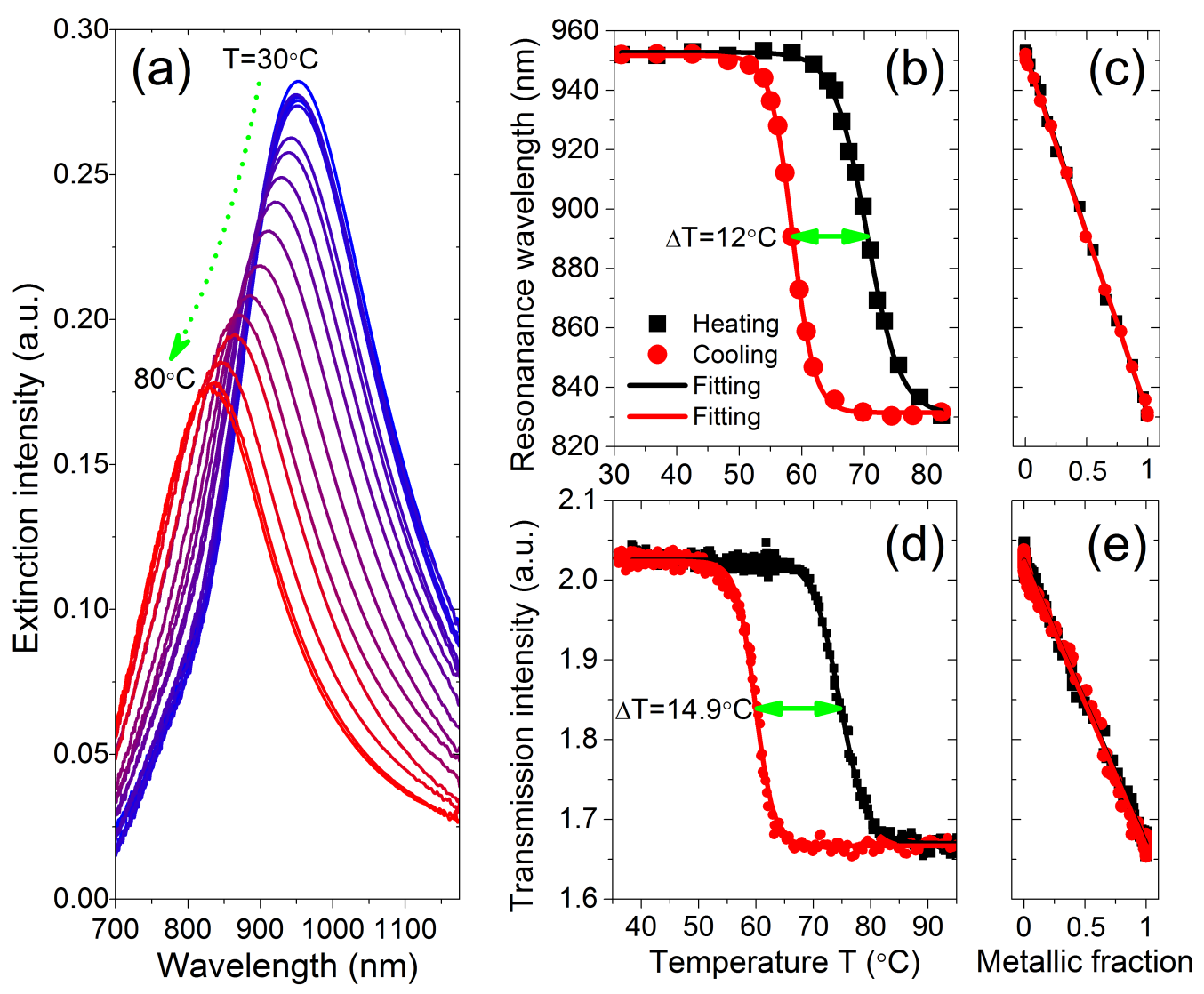

Figure 3: Hysteretic response of localized surface plasmon resonance. (a) Temperaturedependent extinction spectra for $\mathrm{Au}$ nanostructures of $130-\mathrm{nm}$ diameter on $\mathrm{VO}_{2}$ through the heating cycle with a temperature increment of $0.5^{\circ} \mathrm{C}$ around the phase-transition temperature. (b) Plasmon resonance hysteresis curve for the same structure extracted from (a). (d) Ensemble transmission hysteresis curve of a $50-\mathrm{nm} \mathrm{VO}_{2}$ film. Here the sample was illuminated by a tungsten lamp and the integrated, unpolarized transmission intensity was recorded as a function of temperature using an InGaAs photodetector. In both (b) and (d), the black squares and red circles are the measured results for heating and cooling cycles, respectively, while the black and red solid lines are sigmoidal functions fits to the measured data. The green double-headed arrows define the thermal hysteresis width for each system. (c) and (e) Plasmon resonance wavelength from (b) and transmission intensity from (d) as a function of the $\mathrm{VO}_{2}$ effective metallic fraction, respectively.

\section{Persistent optical tuning of LSPR using successive UV-light pulses.}


Apart from the external temperature, UV-light pulses can be used to trigger the insulator-tometal transition from the prepared initial state because $\mathrm{VO}_{2}$ has strong absorption in the UV range, as indicated by the large imaginary component of its dielectric function. ${ }^{36}$ Successive UVillumination pulses also increase the areal density of the metallic phase in $\mathrm{VO}_{2}$, gradually changing the average dielectric response of the film and consequently altering the persistent tuning characteristic of the LSPR. After a sufficiently long illumination time, the metallic nanopuddles connect percolatively and leave the $\mathrm{VO}_{2}$ film in a pure metallic phase.

The hysteresis during the $\mathrm{VO}_{2}$ phase transition allows for persistent tuning of the $\mathrm{Au}$ nanostructure LSPR when the external temperature is biased within the temperature range where strong electron-electron correlation exists. To demonstrate this effect, we prepared the $\mathrm{VO}_{2}$ film in a strongly correlated metal state by maintaining the array and its substrate at a sequence of constant temperatures. The system was then illuminated by successive UV-light pulses either with a constant power density and varying pulse durations or with different power densities at constant pulse duration (varying fluence). After each such pulse sequence, we measured the extinction spectrum of the Au nanodisk array. Control experiments were also performed at two temperatures well below and above the critical temperature of $\mathrm{VO}_{2}$, and the corresponding extinction spectra were recorded for comparison with those collected at temperatures within the strongly correlated range. To verify that the plasmon resonance shift observed in Au nanodisks was due to the altered dielectric response of $\mathrm{VO}_{2}$, spectroscopic ellipsometry ${ }^{39}$ was used to characterize a bare $\mathrm{VO}_{2}$ film of the same thickness $(50 \mathrm{~nm})$ and the change in dielectric response was measured using the same UV-illumination protocol used for the hybrid plasmonic system. The dielectric function of $\mathrm{VO}_{2}$ was extracted by fitting the ellipsometric data with a sum of one Gaussian and one Lorentz oscillator under a global-minimization algorithm. 
Figure 4a shows how the plasmon resonance is modulated by UV illumination for different pulse durations at constant power density $\left(90 \mathrm{~mW} / \mathrm{cm}^{2}\right)$ while keeping the hybrid system at $64{ }^{\circ} \mathrm{C}$ and $68{ }^{\circ} \mathrm{C}$, respectively. The LSPR wavelength extracted from each extinction spectrum as a function of total illumination time exhibits a substantial blue-shift after the initial few pulses, followed by a gradual saturation in the resonance wavelength for much longer pulse durations. Such behavior contrasts sharply with results from the control experiments performed at $27{ }^{\circ} \mathrm{C}$ (not shown) and $77{ }^{\circ} \mathrm{C}$, in which the LSPR wavelength shows no sensitivity whatsoever to the UV illumination. Figure $4 \mathrm{~b}$ shows similar results when the LSPR wavelength is modulated by UV-light pulses of constant duration (10 sec) but with various power densities: A large blue-shift and subsequent resonance saturation also occur when gradually increasing the power density of single UV-light pulses. The inset in Figure $4 \mathrm{~b}$ plots the dispersive refractive index of the bare $\mathrm{VO}_{2}$ film extracted from spectroscopic ellipsometry as a function of the UV-illumination power density at a constant temperature of $65{ }^{\circ} \mathrm{C}$, which reveals a gradual decrease in the refractive index of $\mathrm{VO}_{2}$ in the wavelength range of interest and accounts for the observed blue-shift in the LSPR of Au nanostructures.

In analogy to the general definition of modulation depth for silicon photonic modulators, ${ }^{40}$ here we define the extinction ratio $10 \log \left(I_{0} / I_{\mathrm{UV}}\right)$ as the modulation depth for the $\mathrm{Au} / \mathrm{VO}_{2}$ hybrid heterostructure, where $I_{0}$ and $I_{\mathrm{UV}}$ are the extinction intensities at a specific plasmonic wavelength for the system thermally latched within the phase transition region of $\mathrm{VO}_{2}$ before and after a certain period of UV illumination, respectively. The inset of Figure 4a shows the measured extinction spectra for the $\mathrm{Au}$ nanodisks of $175-\mathrm{nm}$ diameter on the $\mathrm{VO}_{2}$ film before and after 120-sec, $90-\mathrm{mW} / \mathrm{cm}^{2} \mathrm{UV}$ illumination under the bias temperature of $64{ }^{\circ} \mathrm{C}$, from which the modulation depth at the plasmonic wavelength $1029 \mathrm{~nm}$ is calculated to be $1.79 \mathrm{~dB}$. Note that 
this value might be underestimated because in the extinction calculation the transmission of each $\mathrm{Au}$ nanodisk array was normalized with respect to the transmitted light through a bare $\mathrm{VO}_{2}$ area in close proximity to the array. This implies that the extinction contribution from the $\mathrm{VO}_{2}$ itself has not been taken into account in the calculation of modulation depth.
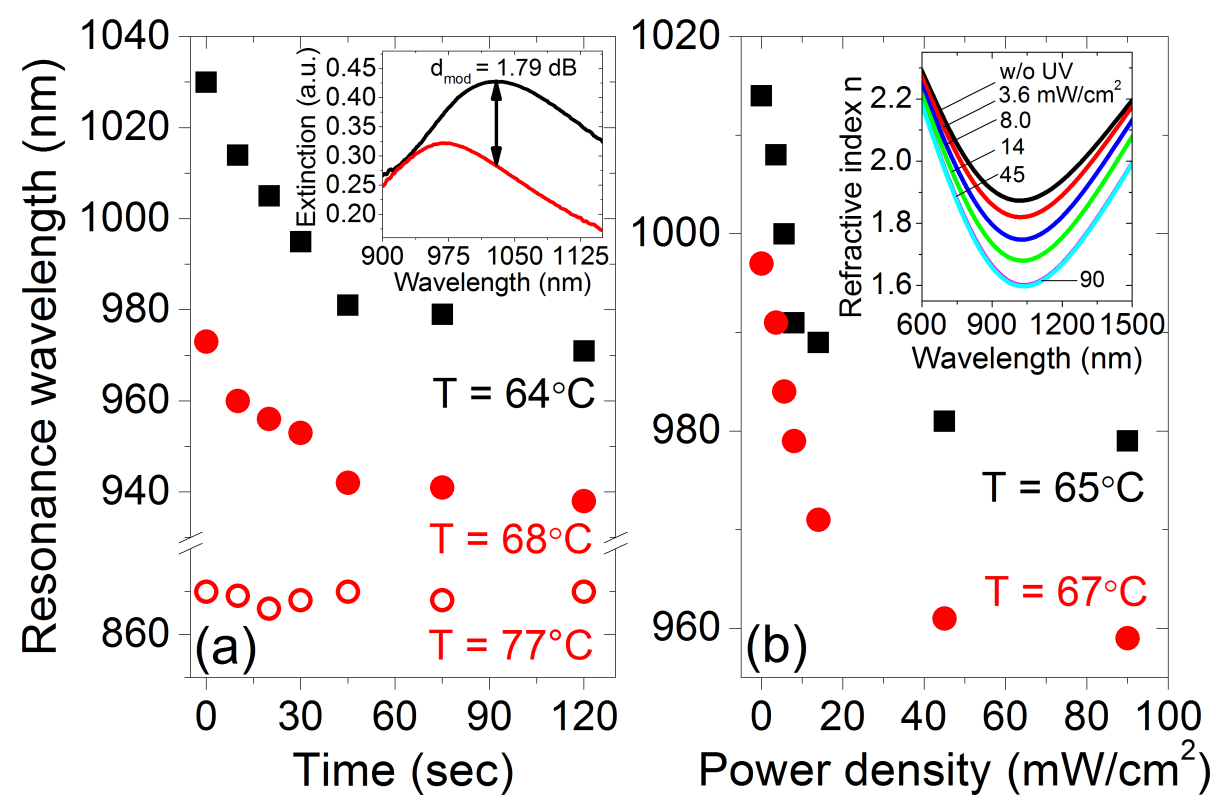

Figure 4: Persistent optical tuning of LSPR by UV-light pulses. (a) Plasmon resonance wavelength of the $\mathrm{Au}$ nanodisks of $175-\mathrm{nm}$ diameter on the $\mathrm{VO}_{2}$ film as a function of the total UV illumination time with a constant power density of $90 \mathrm{~mW} / \mathrm{cm}^{2}$ while the whole sample was thermally latched at two different temperatures within the phase transition region, $64{ }^{\circ} \mathrm{C}$ (black squares) and $68^{\circ} \mathrm{C}$ (red circles), and also above $\left(77^{\circ} \mathrm{C}\right.$, red empty circles). Each of the first three pulses has a duration of $10 \mathrm{sec}$, followed by another two pulses of $15 \mathrm{sec}$, respectively, and the last one of $45 \mathrm{sec}$. Inset shows the measured extinction spectra for the sample latched at $64{ }^{\circ} \mathrm{C}$ before (black) and after 120-sec UV illumination (red), and the modulation depth (i.e. extinction ratio) at a wavelength of $1029 \mathrm{~nm}$ is calculated to be $1.79 \mathrm{~dB}$. (b) Resonance shift for the same structure as a function of the power density of each UV-light pulse of $10 \mathrm{sec}$ duration. Inset shows the dispersive refractive index for a $50 \mathrm{~nm}$ bare $\mathrm{VO}_{2}$ film illuminated at the same conditions as above.

\section{Evolution traces of LSPR registering the initial temperature states.}


In a temperature-controlled memristive device driven by the $\mathrm{VO}_{2}$ phase transition, the resistance takes different paths for cooling and heating cycles; in particular, the time evolution of the resistance depends strongly on the bias temperature from which the cooling cycle starts. ${ }^{41}$ This means that the initial temperature states or the associated information stored in the system can be retrieved by monitoring the evolving path of the system resistance. More generally, in a memristive metal/oxide/metal nanodevice, this corresponds to the hysteretic response of I-V curves in which the applied voltage and resultant current follow a highly nonlinear relationship and the evolutionary history of the current-voltage relationship depends on the initial bias voltage. ${ }^{42,43}$

The optical-frequency, plasmon memory resonance described in this experiment exhibits features analogous to such memristive behavior. Figure 5a plots LSPR wavelength as a function of temperature during the cooling cycle starting from different bias temperatures. Each such evolutionary path is unique, yielding a characteristic parameter pair - thermal hysteresis width and resonance shift - for each bias temperature (Figure 5b). The unique evolution of the LSPR can also be observed by plotting the plasmon resonance wavelengths (extracted from Figure 5a for each different initial temperature and heating cycle) as a function of the effective metallic fraction of $\mathrm{VO}_{2}$ (Figure 5c). The strong similarity between a memristive response ${ }^{41-43}$ and the behavior of the hybrid plasmonic system further supports the idea that an optical-frequency plasmon resonance of the metallic nanostructure can store and read out the "memory" of the initial hybridized state in a broad range of phase-transforming materials like $\mathrm{VO}_{2}$. 

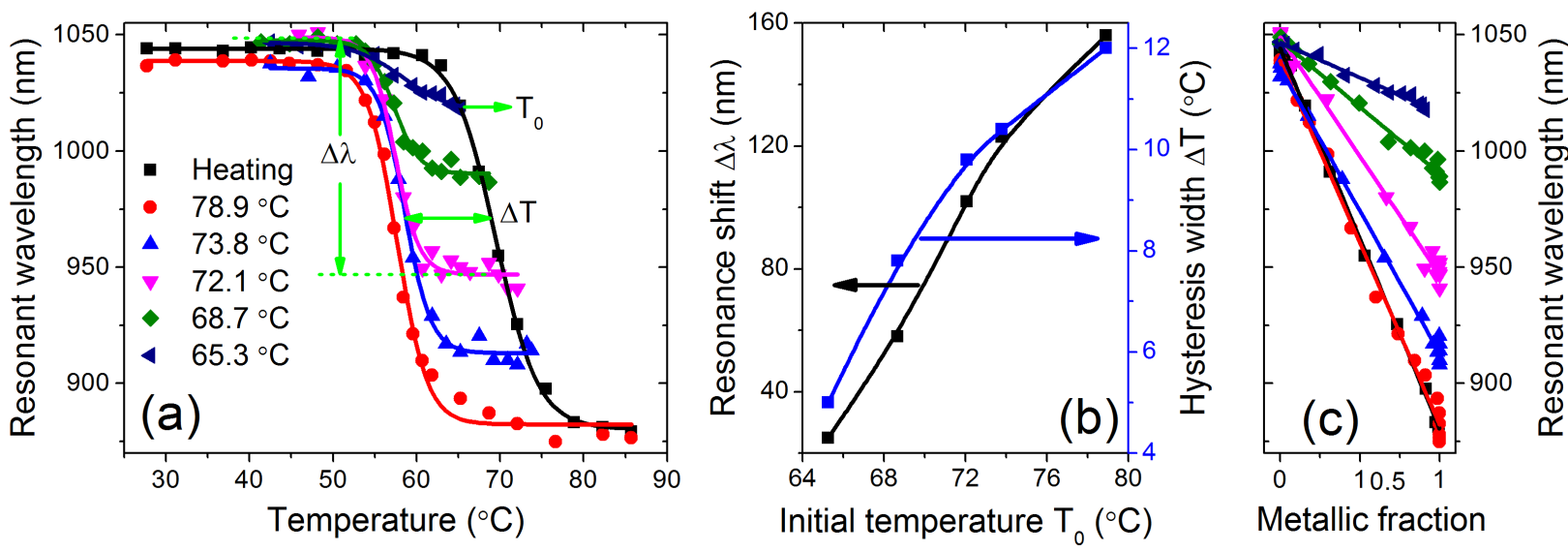

Figure 5: Temperature evolution of LSPR retracing the initial temperature states. (a) LSPR wavelength evolutions for 175 -nm-diameter $\mathrm{Au}$ nanodisks on $\mathrm{VO}_{2}$ through cooling cycles starting from different initial temperatures $\mathrm{T}_{0}$ (red circles to dark-blue left-triangles). The black squares are the resonance positions through the heating cycle. The quantities $\Delta \mathrm{T}$ and $\Delta \lambda$ represent the thermal hysteresis width and the resonance shift amplitude for each initial state of the film, with results shown in (b) as a function of initial bias temperature $\mathrm{T}_{0}$. Solid lines in (a) are sigmoidal fits to the measured data points while black and blue solid lines in (b) are guides to the eye. The plasmon resonance wavelength extracted from (a) for different initial temperatures and heating cycles are re-plotted in (c) as a function of the effective metallic fraction of the $\mathrm{VO}_{2}$.

\section{Discussion}

The hysteretic nature of the first-order phase transition in $\mathrm{VO}_{2}$ is the key to realizing the persistent plasmon resonance tuning in practice. In this experimental configuration a bias temperature somewhere in the transition region from insulator to metal, that is, between $60^{\circ} \mathrm{C}$ and $80^{\circ} \mathrm{C}$ (Figure 4), initializes the $\mathrm{VO}_{2}$ in a strongly correlated state. The UV-modulation experiments reveal that the LSPR of Au nanostructures is "pinned" at a wavelength defined by the initial state of the $\mathrm{VO}_{2}$ film and the duration of illumination. Successive UV-light pulses drive the LSPR to a new wavelength as more $\mathrm{VO}_{2}$ domains are switched from insulating to 
metallic. The hysteretic response of this hybridized heterostructure after the triggering impulse fades away (as demonstrated in Figure 5) is then essential for the long-lasting memory of the UV-light induced change which is "imprinted" into the LSPR frequency.

As noted in the discussion of Figure 5, this LSPR memory effect in metal nanostructures resembles in some ways the memory capacitance and memristive responses of $\mathrm{VO}_{2}$ in memory metamaterials $^{44}$ and phase-transition-based memory devices. ${ }^{41}$ However, the plasmon memory resonance observed here at optical to near-infrared frequencies is based on the hybrid response of the $\mathrm{Au}$ nanodisks and the hysteretic response of $\mathrm{VO}_{2}$. This is distinctly different from the metamaterial memristive system ${ }^{44}$ that exploits the hysteresis in $\mathrm{VO}_{2}$ dielectric constants at terahertz or microwave frequencies. Finally, it should be noted that although UV pulses of a few tens of seconds were used in this study, the phase transition dynamics of $\mathrm{VO}_{2}$ is not intrinsically restricted to slow timescales. The absorbed UV light in the present experiment causes both transient local heating and electronic excitation, leading to the formation of new metallic nanopuddles in the insulating $\mathrm{VO}_{2}$ host and driving the film toward completed formation of the metallic, rutile phase. If the UV energy density is high enough to cause the transition to occur by local heating, the reversion from the rutile metallic state to the insulating state could require nanoseconds to microseconds. If, on the other hand, the UV light pulse is ultrashort ${ }^{26}$ and the transition proceeds primarily through electronic excitation to the recently observed metallic monoclinic state, ${ }^{45-47}$ the complete transition cycle could then occur on a picosecond time scale without a structural phase transition, making it possible to consider practical applications. Another potential route to an ultrafast device using the hybrid material system demonstrated here would be by electron injection initiated by resonant excitation of a noble-metal nanoparticle. ${ }^{27}$ 
The plasmon memory resonance effect can be generalized in a number of ways. Because the critical temperature and the slope of the hysteresis curve for a $\mathrm{VO}_{2}$ film can be substantially altered by impurity doping, ${ }^{48}$ electrical bias,$^{49}$ charge injection ${ }^{27}$ or substrate-induced strain,,$^{50}$ even for the ultrafast laser-driven phase transition, ${ }^{51}$ a plasmon memory device can in principle be operated at lower temperatures, and over a broader temperature range, than what is demonstrated here. Conversely, since the initial strongly correlated metal state can be prepared by applied bias voltage, charge injection, strain or laser illumination, a plasmon memory device can be designed to operate at a constant temperature. The thermal hysteresis width of the IMT in $\mathrm{VO}_{2}$ can be altered by changing fabrication parameters to yield differing grain sizes in a film, ${ }^{23}$ ordered arrays of nanostructures ${ }^{30}$ or strained one-dimensional nanostructures. ${ }^{24}$ Flexible control over both the transition temperature and hysteresis width of the $\mathrm{VO}_{2}$ also allows for stable modulation of the plasmon resonance wavelength.

Other nanostructure configurations can easily be imagined: For example, one could imagine a double-layer lithography ${ }^{32}$ procedure in which one would remove all the $\mathrm{VO}_{2}$ outside the range of the plasmon near-field, leaving a double-layer $\mathrm{Au} / \mathrm{VO}_{2}$ heterostructure array with a defined pitch. Finally, since the phase transformation in $\mathrm{VO}_{2}$ is associated with strong electron-electron correlations, the plasmon memory resonance offers a unique opportunity to monitor the interaction between the collective oscillation of free electrons in the surface plasmons and strongly correlated electrons during the interband transitions in $\mathrm{VO}_{2}{ }^{52}$

Although these experiments employed $\mathrm{Au}$ nanostructure arrays, the behavior observed here should also occur in individual metal nanostructures, and be observable using single-particle spectroscopy to monitor the plasmon resonance shift. In fact, single-particle studies could explore the metal-to-insulator phase-transition on the nanoscale by monitoring electromagnetic 
near fields associated with localized surface plasmons. For example, an individual metal nanostructure used as a nanoprobe could detect coexisting insulating and metallic phases in $\mathrm{VO}_{2}$ and the divergence of the real dielectric function near the onset of the insulator-to-metal transition.

\section{Conclusion}

We have demonstrated a novel plasmon memory resonance by hybridizing the surface plasmon resonance in metallic nanostructures with the strong electron-electron correlation effects in a phase-changing $\mathrm{VO}_{2}$ thin film. Analogous to the memristive response of $\mathrm{VO}_{2}$, this plasmon memory resonance exhibits persistent tuning of the resonance frequency when illuminated by ultraviolet light, in contrast to plasmon modulation schemes using conventional active media. The temperature and temporal evolution of the observed memplasmon resonance depends critically on the correlated initial state of the $\mathrm{VO}_{2}$, and thus enables reversible operation of the device to re-initialize the hybrid plasmonic memory material.

This memory effect on the resonance frequency of a plasmonic nanostructure due to the strong electron-electron correlations in $\mathrm{VO}_{2}$ exhibits an optical analog of memory circuit devices, the plasmon memory resonance, but now in the optical to near-infrared range rather than the microwave and $\mathrm{THz}$ range. ${ }^{41-43}$ Nanoscale integration of plasmonic structures with phasechanging materials such as $\mathrm{VO}_{2}$ could enable further miniaturization of an entire class of memory devices for next-generation nanophotonic circuitry - simply because such devices would work at shorter wavelengths than $\mathrm{THz}$ or microwave-based devices. The dual-excitation scheme presented in this work could potentially be extended to other hybrid plasmonic/SECM systems, incorporating the controlled metal-to-insulator phase transition in $\mathrm{NdNiO}_{3}^{53}$, colossal magnetoresistance in perovskite materials like $\mathrm{LaMnO}_{3}{ }^{54}$ and $\mathrm{Pr}_{0.7} \mathrm{Ca}_{0.3} \mathrm{MnO}_{3} .{ }^{55}$ 
Combined with novel nanostructure architectures ${ }^{32}$ and excitation schemes, ${ }^{56}$ this proof-ofconcept demonstration has a significant impact on the possibilities for transferring the basic concepts in conventional circuits - such as memristive behaviour and memory capacitance - to nanoscale optical devices operating in the visible and near-infrared regimes.

\section{Acknowledgements}

DYL acknowledges support from the Hong Kong Polytechnic University (1-ZVCG). YS and SM acknowledge support from the United Kingdom Engineering and Physical Sciences Research Council and the Leverhulme Trust Foundation. KA and RFH acknowledge support from the National Science Foundation (ECE-0801980); sample nanofabrication and characterization at Vanderbilt University used facilities renovated and upgraded with support from the National Science Foundation (ARI-R2 DMR-0963361). FL acknowledges support from the European Regional Development Fund (CEITEC, CZ.1.05/1.1.00/02.0068).

\section{Corresponding Author}

Correspondence should be addressed to DYL dylei@ polyu.edu.hk. 


\section{References}

(1) Maier, S. A. Plasmonics: Fundamentals and Applications; Springer: New York, 2007.

(2) Bozhevolnyi, S. I.; Volkov, V. S.; Devaux, S.; Laluet, J.-Y.; Ebbesen, T. W. Channel plasmon subwavelength waveguide components including interferometers and ring resonators. Nature 2006, 440, 508.

(3) Liu, Z.; Steele, J. M.; Srituravanich, W.; Pikus, Y.; Sun, C.; Zhang, X. Focusing surface plasmons with a plasmonic lens. Nano Lett. 2005, 5, 1726-1729.

(4) Müller, J.; Sönnichsen, C.; von Poschinger, H.; von Plessen, G.; Klar, T. A.; Feldmann, J. Electrically controlled light scattering with single metal nanoparticles. Appl. Phys. Lett. 2002, 81, 171.

(5) Dickens, M. J.; Sweatlock, L. A.; Pacifici, D.; Lezec, H. J.; Bhattacharya, K.; Atwater, H. A. Electrooptic modulation in thin film barium titanate plasmonic interferometers. Nano Lett. 2008, 8, 4048-4052.

(6) Neutens, P.; Van Dorpe, P.; De Vlaminck, I.; Lagae, L.; Borghs, G. Electrical detection of confined gap plasmons in metal-insulator-metal waveguides. Nat. Photon. 2009, 3, 283.

(7) Suh, J. Y.; Donev, E. U.; Lopez, R.; Feldman, L. C.; Haglund, R. F. Modulated optical transmission of subwavelength hole arrays in metal- $\mathrm{VO}_{2}$ films. Appl. Phys. Lett. 2006, 88, 133115.

(8) Lei, D.Y.; Appavoo, K.; Sonnefraud, Y.; Haglund, R, F. Jr.; Maier, S. A. Single-particle plasmon resonance spectroscopy of phase transition in vanadium dioxide. Opt. Lett. 2010, 35, 3988.

(9) Ren, F.; Wang, X.; Wang, A. X. Thermo-optic modulation of plasmonic bandgap on metallic photonic crystal slab. Appl. Phys. Lett. 2013, 102, 181101.

(10) Temnov, V. V.; Armelles, G.; Woggon, U.; Guzatov, D.; Cebollada, A.; Garcia-Martin, A.; Garcia-Martin, J.-M.; Thomay, T.; Leitenstorfer, A.; Bratschitsch, R. Active magnetoplasmonics in hybrid metal-ferromagnet structures. Nat. Photon. 2010, 4, 107.

(11) Belotelov, V. I.; Akimov, I. A.; Pohl, M.; Kotov, V. A.; Kasture, S.; Vengurlekar, A. S.; Gopal, A. V.; Yakovlev, D. R.; Zvezdin, A. K.; Bayer, M. Enhanced magneto-optical effects in magnetoplasmonic crystals. Nat. Nanotechnol. 2011, 6, 370. 
(12) Bonanni, V.; Bonetti, S.; Pakizeh, T.; Pirzadeh, Z.; Chen, J.; Nogués, J.; Vavassori, P.; Hillenbrand, R.; Åkerman, J.; Dmitrive, A. Designer magnetoplasmonics with nickel nanoferromagnets. Nano Lett. 2011, 11, 5333-5338.

(13) Chin, J. Y.; Steinle, T.; Wehlus, T.; Dregely, D.; Weiss, T.; Belotelov, V. I.; Stritzker, B.; Giessen, H. Nonreciprocal plasmonics enables giant enhancement of thin-film Faraday rotation. Nat. Commun. 2013, 4, 1559.

(14) MacDonald, K. F.; Sámson, Z. L.; Stockman, M. I.; Zheludev, N. I. Ultrafast active plasmonics. Nat. Photon. 2009, 3, 55.

(15) Pacific, D.; Lezec, H. J.; Atwater, H. A. All-optical modulation by plasmonic excitation of CdSe quantum dots. Nat. Photon. 2007, 1, 402.

(16) Utikal, T.; Stockman, M. I.; Heberle, A. P.; Lippitz, M.; Giessen, H. All-optical control of the ultrafast dynamics of a hybrid plasmonic system. Phys. Rev. Lett. 2010, 104, 113903.

(17) Wurtz, G. A.; Pollard, R.; Hendren, W.; Wiederrecht, G. P.; Podolskiy, V. A.; Zayats, A. $\mathrm{V}$. Designed ultrafast optical nonlinearity in a plasmonic nanorod metamaterial enhanced by nonlocality. Nat. Nanotechnol. 2011, 6, 107.

(18) Abb, M.; Albella, P.; Aizpurua, J.; Muskens, O. L. All-optical control of a single plasmonic nanoantenna-ITO hybrid. Nano Lett. 2011, 11, 2457-2463.

(19) Zhu, Y.; Hu, X.; Fu, Y.; Yang, H.; Gong, Q. Ultralow-power and ultrafast all-optical tunable plasmon-induced transparency in metamaterials at optical communication range. Sci. Rep. 2013, 3, 2338.

(20) Hoessbacher, C.; Fedoryshyn, Y.; Emboras, A.; Melikyan, A.; Kohl, M.; Hillerkuss, D.; Hafner, C.; Leuthold, J. The plasmonic memristor: a latching optical switch. Optica 2014, 1, 198-202.

(21) Wuttig, M.; Yamada, N. Phase-change materials for rewriteable data storage. Nat. Mater. 2007, 6, 824 .

(22) Morin, F. J. Oxides which show a metal-to-insulator transition at the Neel temperature. Phys. Rev. Lett. 1959, 3, 34-36.

(23) Suh, J. Y.; Lopez, R.; Feldman, L. C.; Haglund, R. F. Semiconductor to metal phase transition in the nucleation and growth of $\mathrm{VO}_{2}$ nanoparticles and thin films. J. Appl. Phys. 2004, 96, 1209. 
(24) Wei, J.; Wang, Z.; Chen, W.; Cobden, D. H. New aspects of the metal-insulator transition in single-domain vanadium dioxide nanobeams. Nat. Nanotech. 2009, 4, 420.

(25) Qazilbash, M. M.; Li, Z. Q.; Podzorov, V.; Brehm M.; Keilmann, F.; Chae, B. G.; Kim, H. T.; Basov, D. N. Electrostatic modification of infrared response in gated structures based on $\mathrm{VO}_{2}$. Appl. Phys. Lett. 2008, 92, 241906.

(26) Cavalleri, A.; Dekorsy, Th.; Chong, H. H. W.; Kieffer, J. C.; Schoenlein, R. W. Evidence for a structurally-driven insulator-to-metal transition in $\mathrm{VO}_{2}$ : A view from the ultrafast timescale. Phys. Rev. B 2004, 70, 161102(R).

(27) Appavoo, K.; Wang, B.; Brady, N. F.; Seo, M.; Nag, J.; Prasankumar, R. P.; Hilton, D. J.; Pantelides, S. T.; Haglund, R. F. Jr. Ultrafast phase transition via catastrophic phonon collapse driven by plasmonic hot-electron injection. Nano Lett. 2014, 14, 1127-1133.

(28) Dickens, M. J.; Aydin, K.; Pryce, I. M.; Sweatlock, L. A.; Boyd, E. M.; Walavalkar, S.; Ma, J.; Atwater, H. A. Frequency tunable near-infrared metamaterials based on $\mathrm{VO}_{2}$ phase transition. Opt. Express 2009, 17, 18330.

(29) Seo, M.; Kyoung, J.; Park, H.; Koo, S.; Kim, H.-S.; Bernien, H.; Kim, B. J.; Choe, J. H.; Ahn, Y. H.; Kim, H.-T.; Park, N.; Park, Q.-H.; Ahn, K.; Kim, D.-S. Active terahertz nanoantennas based on $\mathrm{VO}_{2}$ phase transition. Nano Lett. 2010, 10, 2064-2068.

(30) Appavoo, K.; Lei, D. Y.; Sonnefraud, Y.; Wang, B.; Pantelides, S. T.; Maier, S. A.; Haglund, R. F. Jr. Role of defects in the phase transition of $\mathrm{VO}_{2}$ nanoparticles probed by plasmon resonance spectroscopy. Nano Lett. 2012, 12, 780-786.

(31) Ye, J.; Van Dorpe, P. Plasmonic behaviors of gold dimers perturbed by a single nanoparticle in the gap. Nanoscale 2012, 4, 7205.

(32) Appavoo, K.; Haglund, R. F. Jr. Polarization selective phase-change nanomodulator. Sci. Rep. 2014, 4, 6771.

(33) Qazilbash, M. M.; Brehm, M.; Chae, B. G.; Ho, P.-C.; Andreev, G. O.; Kim, B.-J.; Yun, S. J.; Balatsky, A. V.; Maple, M. B.; Keilmann, F.; Kim, H.-T.; Basov, D. N. Mott transition in $\mathrm{VO}_{2}$ revealed by infrared spectroscopy and nano-imaging. Science 2007, 318, 1750.

(34) Coy, H.; Cabrera, R.; Sepúlveda, N.; Fernández, F. E. Optoelectronic and all-optical multiple memory states in vanadium dioxide. J. Appl. Phys. 2010, 108, 113115. 
(35) Gurvitch, M.; Luryi, S.; Polyakov, A.; Shabalov, A. Nonhysteretic behavior inside the hysteresis loop of VO2 and its possible application in infrared imaging. J. Appl. Phys. 2009, 106, 104504.

(36) Verleur, H. W.; Barker, A. S.; Berglund, C. N. Optical properties of $\mathrm{VO}_{2}$ between 0.25 and $5 \mathrm{eV}$. Phys. Rev. 1968, 172, 788.

(37) Lopez, L.; Feldman, L. C.; Haglund, R. F. Size-dependent optical properties of $\mathrm{VO}_{2}$ nanoparticle arrays. Phys. Rev. Lett. 2004, 93, 177403.

(38) Appavoo, K.; Haglund, R, F. Jr. Detecting nanoscale size dependence in $\mathrm{VO}_{2}$ phase transition using a split-ring resonator metamaterial. Nano Lett. 2011, 11, 1025-1031.

(39) Lei, D. Y.; Kéna-Cohen, S.; Zou, B.; Petrov, P. K.; Sonnefraud, Y.; Breeze, J.; Maier, S. A.; Alford, N. M. Spectroscopic ellipsometry as an optical probe of strain evolution in ferroelectric thin films. Opt. Express 2012, 20, 4419.

(40) Reed G. T.; Mashanovich, G.; Gardes F. Y.; Thomson, D. J. Silicon optical modulators. Nat. Photon. 2010, 4, 518.

(41) Driscoll, T.; Kim, H.-T.; Chae, B.-G.; Di Ventra, M.; Basov, D. N. Phase-transition driven memristive system. Appl. Phys. Lett. 2009, 95, 043503.

(42) Strukov, D. B.; Snider, G. S.; Stewart, D. R.; Williams, R. S. The missing memristor found. Nature 2008, 453, 80 .

(43) Yang, J. J.; Pickett, M. D.; Li, X.; Ohlberg, D. A. A.; Stewart, D. R.; Williams, R. S. Memristive switching mechanism for metal/oxide/metal nanodevices. Nat. Nanotech. 2008, 3, 429.

(44) Driscoll, T.; Kim, H.-T.; Chae, B.-G.; Kim, B.-J.; Lee, Y.-W.; Jokerst, N. M.; Palit, S.; Smith, D. R.; Di Ventra, M.; Basov, D. N. Memory metamaterials. Science 2009, 325, 1518.

(45) Laverock, J.; Kittiwatanakul, S.; Zakharov, A. A.; Niu, Y. R.; Chen, B.; Wolf, S. A.; Lu, J. W.; Smith, K. E. Direct observation of decoupled structural and electronic transitions and an ambient pressure monocliniclike metallic phase of $\mathrm{VO}_{2}$, Phys. Rev. Lett. 2014, 113, 216402.

(46) Morrison, V. R.; Chatelain, R. P.; Tiwari, K. L.; Hendaoui, A.; Bruhacs, A.; Chaker, M.; Siwick, B. J. A photoinduced metal-like phase of monoclinic $\mathrm{VO}_{2}$ revealed by ultrafast electron diffraction, Science 2014, 346, 445-448.

(47) Wegkamp, D.; Herzog, M.; Xian, L.; Gatti, M.; Cudazzo, P.; McGahan, C. L.; Marvel, R. E.; Haglund, R. F.; Rubio, A.; Wolf, M.; Stähler, J. Instantaneous band-gap collapse in 
photoexcited monoclinic $\mathrm{VO}_{2}$ due to photocarrier doping, Physical Review Letters 2014, 113, 216401.

(48) Shibuya, K.; Kawasaki, M.; Tokura, Y. Metal-insulator transition in epitaxial $\mathrm{V}_{1-x} \mathrm{~W} x \mathrm{O}_{2}(0 \leq x \leq 0.33)$ thin films. Appl. Phys. Lett. 2010, 96, 022102.

(49) Yang, Z.; Hart, S.; Ko, C.; Yacoby, A.; Ramanathan, S. Studies on electric triggering of the metal-insulator transition in $\mathrm{VO}_{2}$ thin films between $77 \mathrm{~K}$ and $300 \mathrm{~K}$. J. Appl. Phys. 2011, $110,033725$.

(50) Wu, J.; Gu, Q.; Guiton, B. S.; de Leon, N. P.; Ouyang, L.; Park, H. Strain-induced self organization of metal-insulator domains in single-crystalline $\mathrm{VO}_{2}$ nanobeams. Nano Lett. 2006, 6, 2313-2317.

(51) Pashkin, A.; Kübler, C.; Ehrke, H.; Lopez, R.; Halabica, A.; Haglund, R. F. Jr.; Huber, R.; Leitenstorfer, A. Ultrafast insulator-metal phase transition in $\mathrm{VO}_{2}$ studied by multiterahertz spectroscopy. Phys. Rev. B 2011, 83, 195120.

(52) Ferrara, D. W.; Nag, J.; MacQuarrie, E. R.; Kaye, A. B.; Haglund, R. F. Jr. Plasmonic probe of the semiconductor to metal phase transition in vanadium dioxide. Nano Lett. 2013, 13, 4169-4175.

(53) Asanuma, S., Xiang, P.-H., Yamada, H.; Sato, H.; Inoue, I. H.; Akoh, H.; Sawa, A.; Ueno, K.; Shimotani, H.; Yuan, H.; Kawasaki, M.; Iwasa, Y. Tuning of the metal-insulator transition in electrolyte-gated $\mathrm{NdNiO}_{3}$ thin films. Appl. Phys. Lett. 2010, 97, 142110.

(54) Ramirez, A. P. Colossal magnetoresistance. J. Phys.: Condens. Matter 1997, 9, 81718199 .

(55) Fiebig, M.; Miyano, K.; Tomioka, Y.; Tokura, Y. Visualization of the local insulatormetal transition in $\operatorname{Pr}_{0.7} \mathrm{Ca}_{0.3} \mathrm{MnO}_{3}$. Science, 1998, 280, 1925-1928.

(56) Aouani, H.; Rahmani, M.; Navarro-Cia, M; Maier, S. A. Third-harmonic-upconversion enhancement from a single semiconductor nanoparticle coupled to a plasmonic antenna. Nat. Nanotechnol. 2014, 9, 290-294. 